\title{
OPERATORS SATISFYING CERTAIN GROWTH CONDITIONS. II
}

\author{
B. C. GUPTA
}

\begin{abstract}
It is proved that the condition $w_{\rho}\left[(T-z I)^{-1}\right]=1 / d(z, \sigma(T))$, $w_{\rho}(\cdot)$ being the operator radius of Holbrook, implies the existence of certain eigenvalues and normal eigenvalues for a Hilbert space operator $T$. This extends known results based on a norm condition $(\rho=1)$ and allows a similar extension of various consequences of these results.
\end{abstract}

Let $C_{\rho}(\rho>0)$ denote the set of all operators on a Hilbert space $H$ with unitary $\rho$-dilation in the sense of [1]. According to Holbrook [2] an operator radius of $T$ is defined by

$$
w_{\rho}(T)=\inf \left\{\alpha: \alpha>0 \text { and } \alpha^{-1} T \in C_{\rho}\right\} .
$$

In particular, $w_{1}(T)=\|T\|$ and $w_{2}(T)=|W(T)|$, the numerical radius of $T$. An operator $T$ is called $\rho$-oid if $w_{\rho}(T)=r(T)$, where $r(T)$ is the spectral radius of $T$.

$T$ is said to satisfy condition $G_{k}(n)$ with respect to $X$ if

$$
\left\|(T-z I)^{-1}\right\| \leqslant k / d(z, X)^{n} \quad \text { for all } z \notin X,
$$

where $X$ is a closed set containing the spectrum $\sigma(T)$ of $T, d(z, X)$ is the distance from $z$ to $X$, and $n$ is a positive integer. The condition $G_{1}(1)$ with respect to $\sigma(T)$ is the same as the usual condition $\left(G_{1}\right)$ of [3].

An operator $T$ is said to be of class $M_{\rho}(\rho \geqslant 1)$ if

$$
w_{\rho}\left[(T-z I)^{-1}\right]=1 / d(z, \sigma(T)) ;
$$

equivalently, $(T-z I)^{-1}$ is $\rho$-oid for all $z \notin \sigma(T)$.

Clearly, the class $M_{1}$ consists of all operators which satisfy condition $\left(G_{1}\right)$ and every operator in $M_{\rho}$ satisfies the condition $G_{\rho}(1)$ with respect to $\sigma(T)$. The classes $M_{\rho}(\rho \geqslant 1)$ are contained in the class of all convexoid operators [5] and form a nondecreasing family with respect to $\rho$. For further properties of operators in $M_{\rho}$ we refer to [5] and [6].

Stampfli has proven the following results, respectively, in [3] and [4].

(1) If $T$ satisfies condition $\left(G_{1}\right)$ then $T$ is isoloid, that is, every isolated point of $\sigma(T)$ is an eigenvalue.

(2) If $\sigma(T)$ lies in a $C^{1}$-Jordan curve $\Gamma$ and $T$ satisfies condition $G_{1}(1)$ outside and condition $G_{k}(n)$ inside $\Gamma$ with respect to $\Gamma$, then $T$ is isoloid.

In this note we prove that the same conclusion is obtained for an operator $T$ of class $M_{\rho}$ without imposing any condition on its spectrum. This leads to

Received by the editors August 16, 1974 and, in revised form, August 29, 1975.

AMS (MOS) subject classifications (1970). Primary 47A20, 47A10.

Key words and phrases. $\rho$-dilation, operator radius, isoloid operators, $M_{\rho}$-operators. 
several corollaries extending various known results based on the norm condition $G_{1}(1)$.

In fact, we can prove the following stronger result.

THEOREM 1. If for some $k<\infty$ and $\rho \geqslant 1$,

$$
w_{\rho}\left[(T-z I)^{-1}\right] \leqslant k / d(z, \sigma(T)), \quad z \notin \sigma(T),
$$

then every isolated point $z_{0}$ of $\sigma(T)$ is an eigenvalue.

Proof. We may assume $z_{0}=0$. Let $P=(2 \pi i)^{-1} \int_{C_{R}}(T-z I)^{-1} d z, C_{R}$ being a circle of radius $R$, be the spectral projection corresponding to the isolated point $z_{0}=0$ of $\sigma(T)$. Then $x \in P(H)$ implies

$$
T x=\frac{1}{2 \pi i} \int_{C_{R}} z(T-z I)^{-1} x d z,
$$

so that

$$
\|T x\| \leqslant\|x\| R^{2} \max _{z \in C_{R}}\left\|(T-z I)^{-1}\right\| .
$$

But $\|S\| \leqslant \rho w_{\rho}(S)$ for every operator $S$ and so

$$
\begin{aligned}
\|T x\| & \leqslant\|x\| \rho R^{2} \max _{z \in C_{R}} w_{\rho}\left[(T-z I)^{-1}\right] \\
& =\|x\| \rho K R^{2} \max _{z \in C_{R}}(d(z, \sigma(T)))^{-1}=\|x\| \rho K R
\end{aligned}
$$

for small $R$. Letting $R \rightarrow 0$ we see that $T x=0$. This completes the proof.

The following theorem, which will be used strongly in our corollaries, has been proved by Patel [5], and for the sake of completeness we include its proof here. Recall that $z$ is a normal approximate eigenvalue of $T$ if there exists a sequence $\left\{x_{n}\right\}$ of unit vectors such that $(T-z) x_{n} \rightarrow 0$ and $\left(T^{*}-\bar{z}\right) x_{n}$ $\rightarrow 0$.

THEOREM 2. Let $T \in M_{\rho}$. If $z_{0}$ is a semibare point of $\sigma(T)$, then it is a normal approximate eigenvalue of $T$.

Proof. We may assume $z_{0}=0$. Let $u \neq 0$ be such that $\{z:|z-u|$ $\leqslant|u|\} \cap \sigma(T)=\{0\}$. Then $d(u, \sigma(T))=|u|$ and $w_{\rho}\left[(T-u I)^{-1}\right] \leqslant|u|^{-1}$. Set $S=-u(T-u I)^{-1}$. Since 0 is in the boundary of $\sigma(T), 0$ is an approximate eigenvalue of $T$. If $\left\{x_{n}\right\}$ is a sequence of unit vectors such that $T x_{n} \rightarrow 0$ then $S x_{n}-x_{n} \rightarrow 0$. Since $w_{\rho}(S) \leqslant 1, S \in C_{\rho}$. It follows from [1, Theorem I.11.1] that $\operatorname{Re} V \geqslant 0$ where

$$
V=(\rho-2)\left(I-S^{*}\right)(I-S)+2(I-S) \text {. }
$$

Therefore

$$
|((\operatorname{Re} V) x, y)|^{2} \leqslant((\operatorname{Re} V) x, x)((\operatorname{Re} V) y, y)
$$

for all $x, y$ in $H$.

Taking $x=x_{n}, y=(\operatorname{Re} V) x_{n}$ and noting that $V x_{n} \rightarrow 0$, the above inequality yields $(\operatorname{Re} V) x_{n} \rightarrow 0$ and so $V^{*} x_{n} \rightarrow 0$. Using $(I-S) x_{n} \rightarrow 0$ it follows that $\left(I-S^{*}\right) x_{n} \rightarrow 0$ and hence $T^{*} x_{n} \rightarrow 0$.

Corollary 1. If $T \in M_{\rho}$ and $\sigma(T)$ is connected, then $\operatorname{Re} \sigma(T)=\sigma(\operatorname{Re} T)$. 
Proof. By Theorem 2, every semibare point of $\sigma(T)$ is a normal approximate eigenvalue of $T$ and hence the proof is on the same lines as in Theorem 4 of Berberian [9].

Corollary 2. If $T \in M_{\rho}$ and $\sigma(T)$ is finite, then $T$ is normal.

Proof. Since every point $z_{i}$ of $\sigma(T)$ is isolated, by Theorems 1 and $2, z_{i}$ is a reducing eigenvalue of $T$. Let $E_{i}$ denote the spectral projection corresponding to $z_{i}$. Then $T=\sum_{i=1}^{n} z_{i} E_{i}$ as in [3] and hence $T$ is normal.

COROLlary 3. If $T$ is reduction $M_{\rho}$, that is, every direct summand of $T$ is in $M_{\rho}$, and every point of $\sigma(T)$ is a semibare point, then Weyl's theorem holds for $T$.

Proof. It follows from Theorems 1 and 2 that $T$ is reduction isoloid and each eigenspace of $T$ is reducing. Hence the result is a consequence of Theorem 5.3 of [7].

COROllary 4. If $T$ is reduction $M_{\rho}$ and $\sigma(T)$ is countable, then $T$ is a diagonal operator.

Proof. By virtue of Theorems 1 and 2, the proof is just an imitation of that of Theorem 1 in [8].

Lastly, we remark that all the corollaries of [8] remain valid if the condition $\left(\alpha^{\prime}\right)$ is replaced by condition 'reduction $M_{\rho}$ ' for $T$.

The author is grateful to the referee for useful suggestions to improve the paper and particularly for pointing out the present direct proof of Theorem 1.

\section{REFERENCES}

1. B. Sz-Nagy and C. Foias, Harmonic analysis of operators on Hilbert space, Masson, Paris; Akad. Kiadó, Budapest, 1967; English rev. transl., North-Holland, Amsterdam; American Elsevier, New York; Akad. Kiadó, Budapest, 1970. MR 37 \#778; 43 \#947.

2. J. A. R. Holbrook, On the power-bounded operators of Sz-Nagy and Foias, Acta Sci. Math. (Szeged) 29 (1968), 299-310. MR 39 \#810.

3. J. G. Stampfli, Hyponormal operators and spectral density, Trans. Amer. Math. Soc. 117 (1965), 469-476; errata, ibid., 550. MR 30 \#3375; 33 \#4686.

4. _ A local spectral theory for operators. III: Resolvents, spectral sets and similarity, Trans. Amer. Math. Soc. 168 (1972), 133-151. MR 45 \#4182.

5. S. M. Patel, On some classes of operators associated with operator radii of Holbrook, 39th Annual Conf. of Indian Math. Soc., Jadavpur University, Jadavpur, 1973.

6. S. M. Patel and B. C. Gupta, Operators satisfying certain growth conditions, Proc. Amer. Math. Soc. 53 (1975), 341-346.

7. S. K. Berberian, The Weyl spectrum of an operator, Indiana Univ. Math. J. 20 (1970/71), 529-544. MR 43 \#5344.

8. Some conditions on an operator implying normality. II, Proc. Amer. Math. Soc. 26 (1970), 277-281. MR 42 \#884.

9. Conditions on an operator implying $\operatorname{Re} \sigma(T)=\sigma(\operatorname{Re} T)$, Trans. Amer. Math. Soc. 154 (1971), 267-272. MR 42 \#5078.

Department of Mathematics, Sarder Patel University, Vallabh Vidyanagar-388120, GUJARAT, INDIA 